\title{
Early outcome of degenerated self-expandable sutureless aortic prostheses treated with transcatheter valve implantation: A pilot series
}

\author{
Nicolas Amabile, MD, PhD, ${ }^{\mathrm{a}}$ Konstantinos Zannis, MD, ${ }^{\mathrm{b}}$ Aurélie Veugeois, MD, ${ }^{\mathrm{a}}$ and \\ Christophe Caussin, MD, ${ }^{\mathrm{a}}$ Paris, France
}

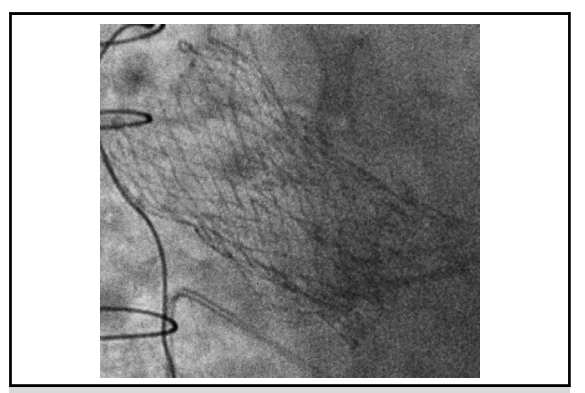

Final result of a valve-in-valve TAVI procedure for treatment of a degenerated SSAD.

\section{Central Message}

The transcutaneous aortic valve implantation procedure is a feasible and safe option for the treatment of degenerated self-expandable sutureless aortic prostheses.

See Editorial Commentary page 1638.
Self-expandable sutureless aortic device (SSAD) implantation may represent an alternative to conventional aortic valve replacement, because it requires shorter cardiopulmonary bypass and crossclamp times and provides a better hemodynamic profile with a larger effective aortic orifice. ${ }^{1}$ These prostheses also, however, may deteriorate with time and require subsequent treatment.

Percutaneous transcatheter aortic valve-in-valve (VIV) implantation is now acknowledged as a valid option for treatment of patients with a failed bioprosthesis who are at high risk with reoperative conventional surgery. ${ }^{2}$ Nevertheless, the benefit of VIV for the treatment of a deteriorated SSAD is unknown. These devices are different from usual aortic conventional surgical prosthesis because they are built over a large nitinol stent structure, have a less rigid ring, and are not tightly attached to the vessel wall (because the valve anchoring is provided by the outer stent radial force), thus giving theoretically less support for an additive device.

The aim of this study was to assess the feasibility and the short-term outcome of the VIV procedure in patients with degenerated SSADs.

From January 2015 to April 2016, we enrolled in this prospective registry all consecutive patients with a degenerated aortic prosthesis who were referred to our center for VIV procedures. All the patients were proposed candidates for a percutaneous transcatheter VIV procedure according to heart team consensus because of some contraindication to conventional aortic valve replacement or high surgical

TABLE 1. Initial clinical characteristics and results after sutureless self-expandable device implantation

\begin{tabular}{|c|c|c|c|c|c|c|c|}
\hline Patient & Sex & STS score & Indication for SSAD & $\begin{array}{c}\text { Creatinine } \\
\text { clearance } \\
\left(\mathbf{m L} / \mathbf{m i n} / \mathbf{m}^{2}\right)\end{array}$ & SSAD type & $\begin{array}{c}\text { Postoperative } \\
\text { MTG (mm Hg) }\end{array}$ & $\begin{array}{c}\text { Postoperative } \\
\text { AR } \\
\end{array}$ \\
\hline 1 & $\mathrm{~F}$ & 4.2 & AS and mitral repair & 34.2 & Perceval M & 5 & Moderate \\
\hline 2 & $\mathrm{~F}$ & 4.9 & AS & 70.2 & Perceval M & 19 & None \\
\hline 3 & $\mathrm{~F}$ & 3.7 & AS & 116.4 & Perceval M & 11 & Mild \\
\hline 4 & M & 2.3 & AS & 80.9 & Perceval L & 23 & Mild \\
\hline 5 & $\mathrm{~F}$ & 3.3 & AS & 65.9 & Perceval M & 12 & None \\
\hline
\end{tabular}

STS, Society of Thoracic Surgeons; SSAD, sutureless self-expandable device; $M T G$, mean transaortic gradient; $A R$, aortic regurgitation; $A S$, aortic stenosis; $M$, medium; $L$, large. 
TABLE 2. Baseline and procedural characteristics of patients

\begin{tabular}{|c|c|c|c|c|c|c|c|c|c|c|c|}
\hline Patient & Age (y) & $\begin{array}{c}\text { Delay } \\
\text { between } \\
\text { AVR and } \\
\text { TAVI (mo) }\end{array}$ & $\begin{array}{c}\text { Degeneration } \\
\text { mode }\end{array}$ & $\begin{array}{c}\text { Pre-TAVI } \\
\text { MTG } \\
(\mathrm{mm} \mathrm{Hg})\end{array}$ & $\begin{array}{c}\text { Pre-TAVI } \\
\text { peak velocity } \\
(\mathrm{m} / \mathbf{s})\end{array}$ & $\begin{array}{c}\text { Pre-TAVI } \\
\text { AR }\end{array}$ & $\begin{array}{l}\text { STS } \\
\text { score }\end{array}$ & TAVI device & $\begin{array}{c}\text { Immediate } \\
\text { MTG } \\
\text { (mm Hg) }\end{array}$ & $\begin{array}{c}\text { 30-d MTG } \\
\text { (mm Hg) }\end{array}$ & 30-d AR \\
\hline 1 & 83.2 & 37.6 & Mixed & 26 & 3.3 & Severe & 9.6 & Evolute-R, 26 mm & 23 & 10 & None \\
\hline 2 & 82.9 & 79.7 & Stenosis & 54 & 4.5 & None & 7.2 & Evolute-R, $26 \mathrm{~mm}$ & 33 & 20 & Mild \\
\hline 3 & 83.8 & 72.7 & Mixed & 21 & 3.1 & Severe & 6 & Evolute-R, $26 \mathrm{~mm}$ & 16 & 8 & None \\
\hline 4 & 80.9 & 65.7 & Stenosis & 45 & 4.2 & None & 4.7 & Evolute-R, $26 \mathrm{~mm}$ & 16 & 19 & Mild \\
\hline 5 & 72.4 & 62.6 & Mixed & 52 & 4.1 & Moderate & 16.3 & CoreValve, $23 \mathrm{~mm}$ & 32 & 15 & None \\
\hline
\end{tabular}

$\overline{A V R}$, Aortic valve replacement; TAVI, transcatheter aortic valve implantation; $M T G$, mean transaortic gradient; $A R$, aortic regurgitation; STS, Society of Thoracic Surgeons.

risk. The patients underwent transcutaneous aortic valve implantation (TAVI) according to the current guidelines and benefited from a subsequent clinical follow-up. Valve Academic Research Consensus 2 criteria were used to assess postprocedural outcomes. ${ }^{3}$ All patients gave their consent before inclusion, and the protocol was approved by our local ethics committee.

During the study period, there were a total of 309 TAVI procedures performed in our center, including 25 patients with VIV procedures and 5 subjects ( $80 \%$ female; median age, 82.9 years; interquartile range [IQR], 2.3 years; median Society of Thoracic Surgeons score, 8.8; IQR, 3.6; median creatinine clearance, $70.2 ; \mathrm{IQR}, 15 \mathrm{~mL} / \mathrm{min} / \mathrm{m}^{2}$ ) with failed SSADs. The median delay between implantation and reintervention was 65.7 months (IQR, 10.1 months).

The initial indication for SSAD was isolated aortic stenosis in an intermediate surgical risk situation in all cases except for that of patient 1 , who underwent a concomitant mitral valve repair. The baseline characteristics of the patients at the time of SSAD implantation are given in Table 1. The median initial Society of Thoracic Surgeons score was 3.7 (IQR, 0.9). The median mean transaortic gradient (MTG) was 45 (IQR, $26 \mathrm{~mm} \mathrm{Hg}$ ), and an

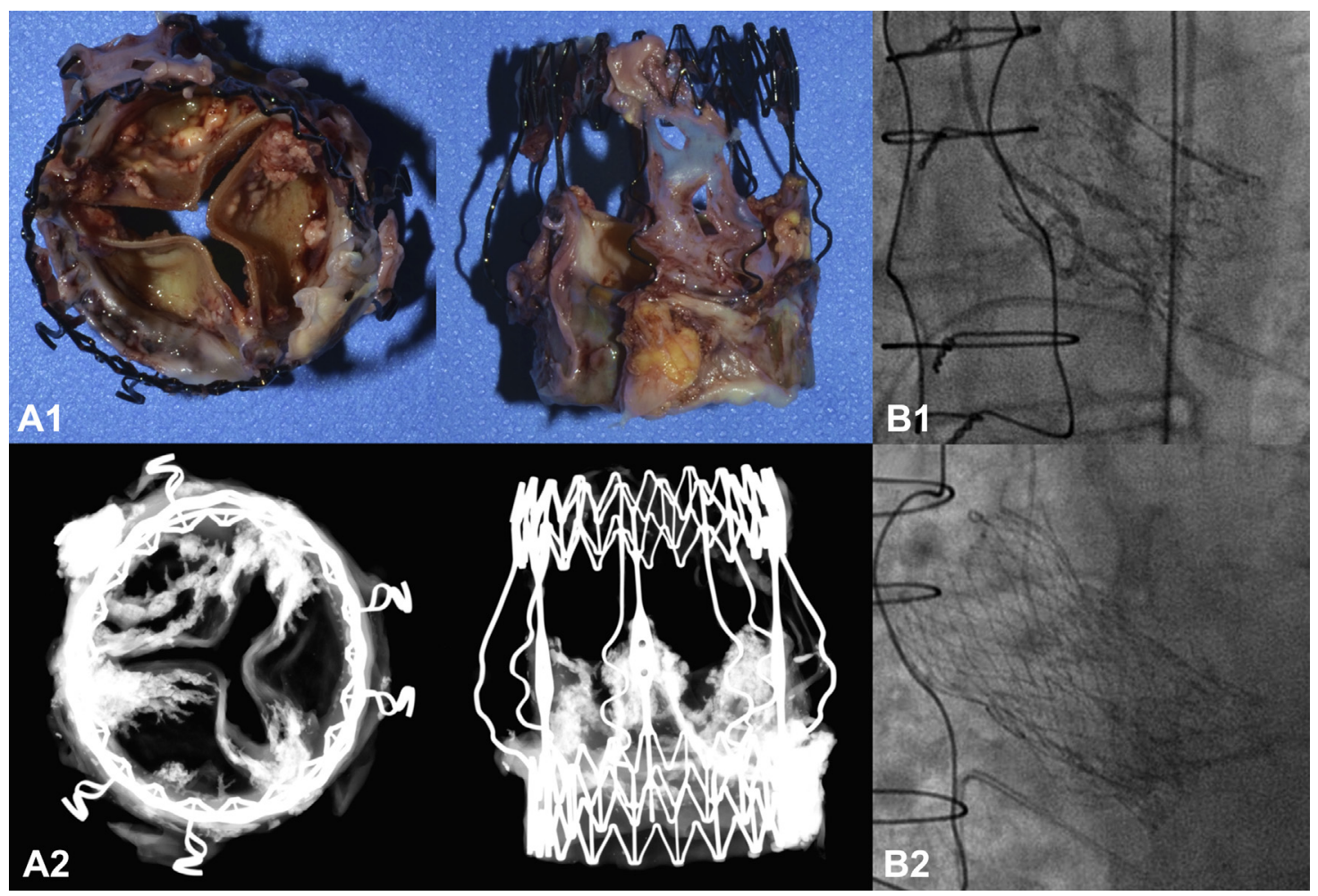

FIGURE 1. A, Postoperative views of an explanted degenerated Perceval self-expandable sutureless aortic valve (LivaNova PLC, London, United Kingdom) depicting severe commissural calcifications. Images show gross pathologic analysis above $\left(A_{1}\right.$ and $\left.A_{2}\right)$ and corresponding radiographic specimen analysis below $\left(A_{3}\right.$ and $\left.A_{4}\right)$. B, Angiographic views of a degenerated self-expandable sutureless aortic device before $\left(B_{1}\right)$ and after $\left(B_{2}\right)$ implantation of a selfexpanding Medtronic Evolute-R device (Medtronic, Minneapolis, Minn) in the valve-in-valve position. 


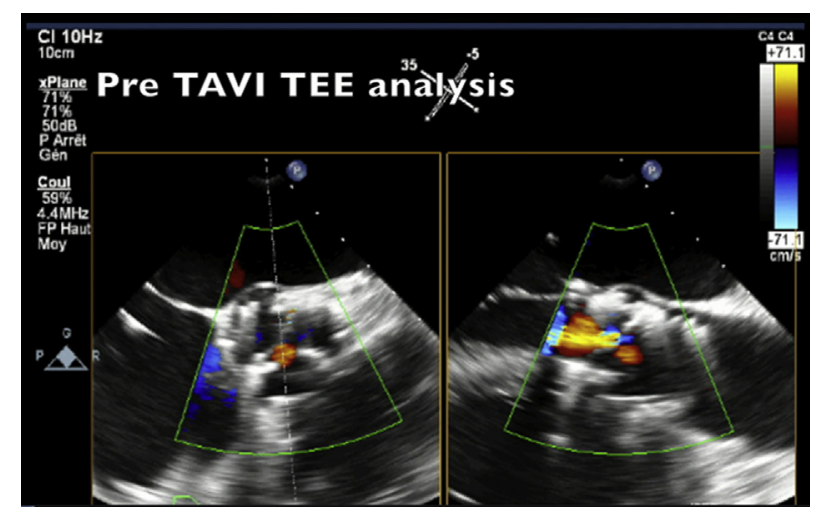

VIDEO 1. Example of a valve-in-valve transcutaneous aortic valve implantation procedure in a degenerated self-expandable sutureless aortic device with a Medtronic Evolute-R device (26 mm; Medtronic, Minneapolis, Minn) through transfemoral access. Video available at: http://www. jtcvsonline.org/article/S0022-5223(16)31051-0/addons.

intraprosthetic moderate regurgitation was reported in 1 patient after SSAD implantation. These 5 patients represented $1.9 \%$ of the 265 SSADs implanted in our center between 2007 and 2016. In the same time frame, 2 patients underwent conventional reoperative aortic valve replacement for degenerated SSADs and another patient died before planned TAVI. Overall, $3 \%$ of our patients with SSADs underwent reintervention for valve deterioration according to the consensus definition. ${ }^{4}$

The clinical and interventional characteristics of the 5 patients at the time of TAVI are given in Table 2. All VIV procedures were performed through transfemoral access with self-expandable devices (Figure 1 and Video 1). Degenerated valve predilation was used in 1 patient. There was no need for a second device in any patient.

The success rate was $100 \%$. There were no device migrations, no deaths, and no periprocedural adverse events (stroke, myocardial infarction, major or life-threatening bleeding events, new pacemaker implantation, coronary ostium occlusion). A mild postprocedural aortic regurgitation was noted in 2 patients.

The 30-day postprocedural clinical course was uneventful in all cases. MTG significantly decreased with time: Median MTG was $45 \mathrm{~mm} \mathrm{Hg}$ (IQR, $26 \mathrm{~mm} \mathrm{Hg}$ ) before the procedure, $24 \mathrm{~mm} \mathrm{Hg}$ (IQR, $16 \mathrm{~mm} \mathrm{Hg}$ ) immediately afterwards, and $14 \mathrm{~mm} \mathrm{Hg}$ (IQR, $9 \mathrm{~mm} \mathrm{Hg}$ ) 30 days after TAVI $(P<.05$ vs baseline for immediate and 30-day postoperative measurements; $P<.05$ for 30 -day vs immediate postoperative measurements; Student paired $t$ test). Comparable results were observed at 90 days.

This pilot series suggests that VIV TAVI procedure is a feasible and safe option for treatment of degenerated SSADs in selected patients. Because these prostheses were initially implanted in these 5 patients for a previous intermediate to high surgical risk (because of the aforementioned potential benefits), the option of a reoperative surgery was not considered. The factors explaining the degeneration process in these subjects were not clearly identified; the post SSAD implantation early hemodynamic data were in line with published results, ${ }^{1}$ and renal function was normal in most patients. Unfortunately, the design of this series does not allow us to draw conclusions regarding the long-term outcome of the patients with implanted SSADs; these (as well as potential risk factors for valve deterioration) will be assessed by larger, multicenter registries.

Our results show that a VIV procedure achieved hemo-

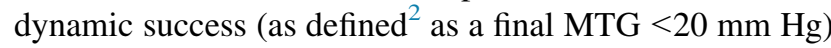
was achieved in $80 \%$ of patients. It should be noted, however, that MTG decreased with time after the procedure, suggesting that the dimensions and effective orifice area of the 2 associated self-expanding devices kept on growing during the first weeks after implantation. From this perspective, our data indicate that the final assessment of VIV result in this particular situation could be deferred. Further data from multicenter series are required to confirm these results.

\section{References}

1. Fischlein T, Meuris B, Hakim-Meibodi K, Misfeld M, Carrel T, Zembala M, et al The sutureless aortic valve at 1 year: a large multicenter cohort study. J Thorac Cardiovasc Surg. 2016;151:1617-26.e4.

2. Dvir D, Webb JG, Bleiziffer S, Pasic M, Waksman R, Kodali S, et al; Valvein-Valve International Data Registry Investigators. Transcatheter aortic valve implantation in failed bioprosthetic surgical valves. JAMA. 2014;312:162-70.

3. Kappetein AP, Head SJ, Génereux P, Piazza N, van Mieghem NM, Blackstone EH et al. Updated standardized endpoint definitions for transcatheter aortic valve implantation: the Valve Academic Research Consortium-2 consensus document; Valve Academic Research Consortium-2. J Thorac Cardiovasc Surg. 2013;145: 6-23.

4. Akins CW, Miller DC, Turina MI, Kouchoukos NT, Blackstone EH Grunkemeier GL, et al; Councils of The American Association for Thoracic Surgery; Society of Thoracic Surgeons; European Assoication for Cardio-Thoracic Surgery; Ad Hoc Liaison Committee for Standardizing Definitions of Prosthetic Heart Valve Morbidity. Guidelines for reporting mortality and morbidity after cardiac valve interventions. J Thorac Cardiovasc Surg. 2008;135:732-8. 